\section{Probability processing and diagnostic search: 20 alternatives, 500 trials*}

\author{
ROBERT G. MILLS $†$ \\ Aerospace Medical Research Laboratory \\ Aerospace Medical Division, Air Force Systems Command \\ Wright-Patterson AFB, Ohio 45433
}

Ss' capabiiities for processing probability information in performing a diagnostic search task similar to troubleshooting is explored. Six Ss searched (queried) among 20 components in a series circuit to isolate a single component failure per trial. Failures were generated according to 10 alternative probability-of-component-failure distributions (D), each with $\mathrm{N}=500$. Prior to performing their search on a trial, Ss predicted the failure for that trial. Of primary interest was the influence of varying $D$ on predictions and search performance. Results indicate that: (1) distributions of predictions tend to match actual $D$ in shape and location but exhibit certain characteristics; (2) probability of correct prediction is low and is influenced by $D$; (3) search strategies are suboptimal but "logical" and to some extent predictable; and (4) in some instances, search strategy appears to be related to predictions.

This study explored Ss' capability to extract and use probabilistic information in a diagnostic search task similar to troubleshooting. In this type of decision task, Ss must identify sets of alternatives based on status information obtained via testing or query.

Ss searched among 20 alternatives (component connections in a series circuit) to determine which component had been failed by $E$. The component failed on each of 500 trials was determined a priori from a hypothetical probability-of component-failure distribution. Before conducting his search on each trial, $\mathbf{S}$ predicted the component failure on that trial.

If $\mathrm{S}$ were able to aggregate and use his accumulated experience from prior trials, the probability distribution of his predictions $(\mathrm{Pc})$ would, over the sequence of 500 trials, approach the corresponding actual probability distribution of failures (D). Furthermore, if $S$ were to use probability information optimally in performing diagnostic search, his search performance would approach that generated by an optimal strategy using $\mathrm{D}$.

of specific interest in the study were the (1) extent that subjective (response) Ds, as reflected by $\mathrm{Pc}$,

\footnotetext{
* The research reported in this paper was sponsored by the Aerospace Medical Research Laboratory, Aerospace Medical Division, Air Force Systems Command. Wright-Patterson AFB, Ohio. This paper is identified as AMRL-TR-71-38. Reprint requests should be sent to the author, AMRL (HES), WPAFB, Ohio 45433 .

tThe author acknowledges the assistance of Beverly Hopkins, Michael A. Bauer, and Charles Rinehart in conducting this research and the critical help provided by Dr. Melvin J. Warrick.
}

match corresponding actual Ds; (2) statistical characteristics of $\mathrm{Pc}$; (3) extent that Ss' search strategies match optimal search strategy; and (4) extent that both $\mathrm{Pc}$ and search performance are influenced by varying D.

\section{APPARATUS}

Indicator/switch modules arranged on a panel in a 4 by 5 matrix comprised a series electrical circuit of Components C1 through C20. Two modules to the right of the component matrix were used for instruction and control. Legends identified component numbers and instructions (e.g., Component 15 ; ESTIM A TE: Press-to-Continue). The panel was $12 \times 8$ in. and was mounted approximately 12 in. in front of $S$.

Circuit status to and including $\mathrm{Ci}$ $(i=1-20$ ) could be determined by "press-to-test" at Ci. A completed circuit through $\mathrm{Ci}$ was indicated by a green light at $\mathrm{Ci}$ when it was pressed. No light indicated a failure at, or prior to, Ci. Failing a component was accomplished by programmed interruption of the voltage signal to that component. Component tests and control responses were automatically recorded.

\section{TASK}

S's task on each trial was divided into two parts, "estimate" and "search." S first predicted the component that would fail on the trial, then initiated search to locate and verify the failed component (the component failing its test and adjacent to one that passed). Verification required that $S$ terminate his search by testing, in sequence, the last operative component and then the failed component. Given Failure $\mathrm{C} 4$, a possible test sequence might be: $\mathrm{C} 8$ (fail) $-\mathrm{C} 3$ (pass) $-\mathrm{C} 5$ (fail) $\mathrm{C} 4$
(fail)-C3 (pass) $-\mathrm{C} 4$ (fail). The last two tests are verification and are not counted in the analysis.

MATERIALS

Component failures were generated according to $10 \mathrm{Ds}, \mathrm{D} 1$ through $\mathrm{D} 10$, of 500 failures each (see Ex of Fig. 1 for the failure probability of each component across D; Fig. 1 is clarified below). D ranged from exponential with $\mathrm{C} 1$ having the highest probability of failure (D1) to exponential with C20 having the highest probability of failure (D10). D9 and D10 were complements of D2 and D1, respectively. Each $\mathrm{D}$ was generated using the Weibull density function with $N=500$ (see Mills \& Bauer, 1971). Order of failure occurrence was randomized across trials.

\section{SUBJECTS}

Six undergraduate university students served as Ss. All were paid volunteers.

\section{PROCEDURE}

The 500 trials required under each $D$ were considered too many to permit testing each $S$ on each $D$. Ss were paired, and each pair completed six of the 10 Ds. Thus, Ss 1 and 2 completed D2, D3, D5, D6, D8, D10; Ss 3 and 4 completed D2, D4, D5, D7, D8, D9; and $\mathrm{Ss} 5$ and 6 completed D1, D3, D6, D7, D8, D9. Equipment difficulties prevented S 6 from completing D7 entirely. This S-D combination is not included in the analysis.

Ss were tested individually in hourly sessions, normally separated by $48 \mathrm{~h}$, completing each $\mathrm{D}$ in three sessions of 150,150 , and 200 trials each. D order was randomized across pairs of Ss and counterbalanced within pairs.

The Ss received an initial instruction period and a practice session of 20 trials. They were instructed to maximize number of correct predictions and minimize total number of search tests per trial. Examples ind icated how long-range troubleshooting costs may be minimized by adopting this approach.

RESULTS AND CONCLUSIONS ${ }^{1}$ Component Failure

Prediction Performance

$N$ for each $D$ has been taken as 500 , although actual $\mathrm{N}$ values varied slightly ( $\mathrm{N}=495-500)$ due to an occasional loss of trial. Ex is used to denote the expected distribution in terms of performance. When $\mathrm{N}=500, \mathrm{Ex}$ is identical to $D$.

Prediction Distributions

Average predicted component $(\mathrm{Pc})$ failure distributions are shown in Fig. 1. Ss forming each average are indicated under Procedure. As may be observed in Fig. $1, \mathrm{Pc}$ tends to vary with Ex in shape and location. The overall accuracy of Pc-Ex matching is indicated by a mean absolute difference between $P(P c)$ and $P(E x)$ 


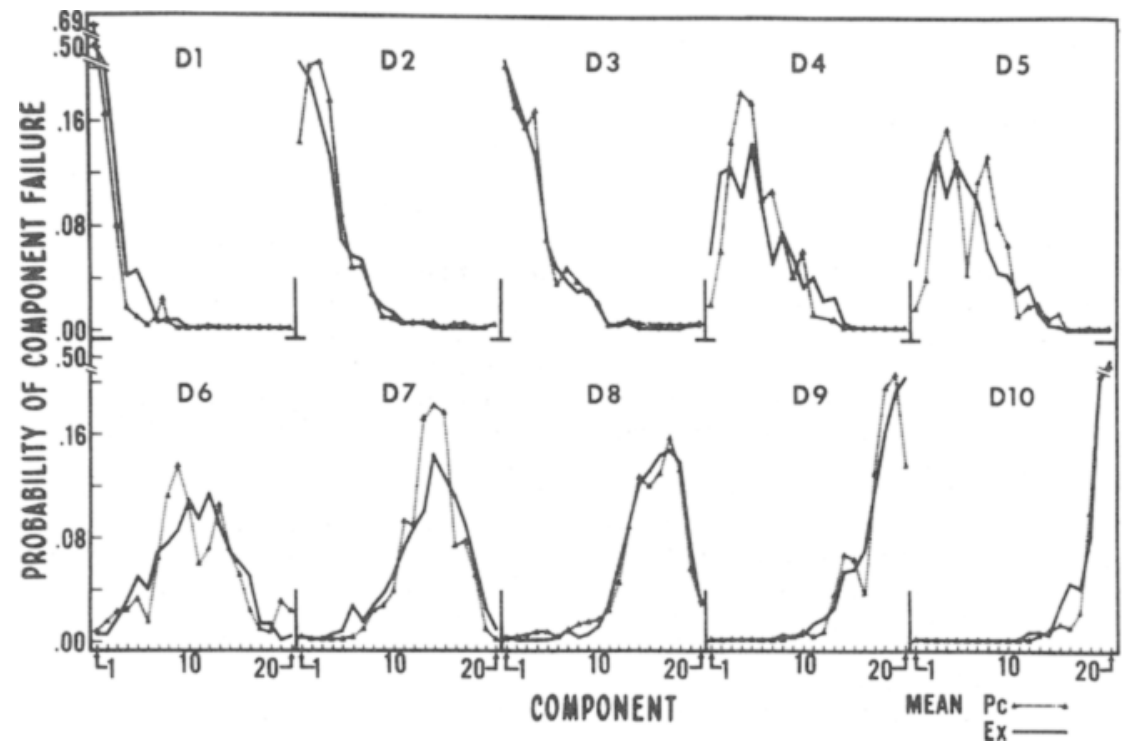

Fig. 1. Pe and Ex probability distributions across $\mathbf{D}$.

collapsed over Ss and D of only .04, with $\mathrm{SD}=.03$, range $=.01$ to .23 , and $\mathrm{N}=439$ (zero categories with zero differences were excluded because such an outcome could result from perfect performance or the unique effect of no failure occurrence).

Despite this matching, however, $\mathrm{Pc}$ exhibits some interesting characteristics. First, Ss are conservative in predicting failures of components at the extremes of the component scale. On $74 \%$ of 35 total $S-D$ combinations, $P c$ underestimated the frequency of the extreme component category (C1 or C20) nearest mean Ex. This is difficult to observe in Fig. 1, but good examples are $\mathrm{Pc}$ for $\mathrm{D} 2$ and $\mathrm{D} 9$ in which $\mathrm{C} 1$ and C 20 failures, respectively, are underestimated. $\mathrm{Pc}$ for $\mathrm{D} 1$ and $\mathrm{D} 10$, however, has overcome this underestimation tendency, suggesting that the effect is frequency-dependent.

Secondly, although $\mathrm{Pc}$ fluctuations are in some cases exaggerated, close examination of Fig. 1 indicates that Pc responds to $E x$ fluctuations that are quite small in terms of P(Ex). D5, for example, has a peak probability at C3 of .14 that changes negatively by .04 to .10 at $\mathrm{C} 4$ and then changes positively by .03 to .13 at C5. Corresponding $\mathrm{Pc}$ peaks appear to be in the correct direction but are exaggerated and shifted away from the $\mathrm{C} 1$ end of the component scale at $\mathrm{C} 4$, C6, and $C 8$, respectively.

Third, there is little difference between $\mathrm{Pc}$ for $\mathrm{D} 1$ and its complement D10 or for D2 and its complement D9, indicating that positional (left, right) effects of scale are not present.

Comparison of $\mathrm{Pc}, \mathrm{Ex}$ Moments

Figure 2 illustrates $\mathrm{Pc}$ and $\mathrm{Ex}$ first moment (mean) and second moment (variance) comparisons for nonaccumulated (NAC) and accumulated (AC) blocks of 20 trials. Under NAC, successive blocks are treated independent of prior blocks. Under AC, each successive block is added to prior blocks. The S's data shown characterize performance of remaining Ss.

Mean $\mathrm{Pc}(\mathrm{MPc})$ tracks mean $\mathrm{Ex}$ (MEX) closely across NAC and AC. Under $A C$, the means are nearly identical at Terminal Block 25. Pc variance (VPc) does not track $\mathrm{Ex}$ variance (VEx) closely over NAC or AC. VPc generally is less than VEx and does not fluctuate nearly as widely. Although often displaced, VPc, under NAC, tends to fluctuate with VEx, but not in comparable magnitude. The exception was D6 which has the largest variance of the $\mathrm{Ds}$ investigated. suggesting a VEx crossover value below which VPc is less than VEx and above which VPc exceeds VEx. Previous results (e.g., Peterson \& Beach, 1967), indicate this relationship may be explained in terms of a model emphasizing frequency of extreme deviations from the mean rather than least squares. Alternatively, however, MEx for D6 is positioned near the center of the component scale which, when combined with a large VEx, may have had a unique effect on $\mathrm{Pc}$.

Ratios of $\mathrm{Pc}$ to $\mathrm{Ex}$ moments computed for each S-D combination indicate $80 \%$ of VPc/VEx, $74 \%$ of SPc/SEx (third moment skewness), and $80 \%$ of $\mathrm{KPc} / \mathrm{KEx}$ (fourth moment kurtosis) ratios were less than 1.0 .

A multiple regression analysis was performed between $\mathrm{Pc}$ and Ex moments for NAC blocks of 50 trials collapsed over Ss and D $(\mathrm{N}=350$ blocks; the same procedure is used for other regression analyses below). Multiple regression equations, multiple correlation coefficients (R), and standard errors of estimate (SE) for $\mathrm{MPc}$ and $\mathrm{VPc}$ are shown below (only nonzero terms are shown). $R$ values for $\mathrm{SPc}(\mathrm{R}=.22)$ and $\mathrm{KPc}(\mathrm{R}=.26)$ were considered too low to make their equations useful here.

$$
\begin{aligned}
\mathrm{MPc}= & .98 \mathrm{MEx}-.05 \mathrm{VEx}+.60 \\
\mathrm{R}= & .98 \\
\mathrm{SE}= & 1.33 \\
\mathrm{VPc}= & .10 \mathrm{MEx}+.83 \mathrm{VEx}-.10 \mathrm{SEx} \\
& +.62 \\
\mathrm{R}= & .49 \\
\mathrm{SE}= & 5.55
\end{aligned}
$$

Additional $\mathrm{Pc}$ characteristics are suggested by the above results. Thus MPc is a predictable and an accurate approximator of $\mathrm{MEx}$. This, however, is not the case for VPc, $S P c$, or $\mathrm{KPc}$. Furthermore, $\mathrm{Pc}$ is less variable, more peaked and less skewed than Ex. Lastly, MEx and VEx are essentially the sole contributors to the variance of $\mathrm{MPc}$ and $\mathrm{VPc}$, respectively.

That $\mathrm{Pc}$ is more peaked than Ex but still approximates $\mathrm{MEx}$ indicates that $\mathrm{Pc}$ overestimates higher Ex frequencies and underestimates lower Ex frequencies. That $\mathrm{Pc}$ is less skewed



Fig. 2. Pc and Ex first and second moments under nonaccumulated (NAC) and accumulated (AC) blocks of 20 trials. Data are for a single $S$ under D8. 
than Ex and underestimates extreme category frequencies indicates that $\mathrm{Pc}$ is shifted slightly away from extreme categories.

Probability of Correct Pc

Probability of correct $\mathrm{Pc}, \mathrm{P}(\mathrm{CPc})$, averaged across $S s$ within $D$ is generally low, but is to some extent influenced by D. For D1 through D10, average $\mathrm{P}(\mathrm{CPc})=.47, .16, .16, .11$, $.10, .09, .12, .14, .16, .39$, respectively. The high $\mathrm{P}(\mathrm{CPc})$ obtained for D1 and D10 is not unexpected because these Ds are nearly binary in form.

\section{DIAGNOSTIC SEARCH} PERFORMANCE

The optimal model employed for evaluating $S$ s' search strategy performed the component test that split the probability of each unresolved set of possible failed components (the known set of components that, prior to a test, contains the failed component) into two equally probable sets. The optimal solution was obtained on a trial-by-trial comparative basis using a computer by having the optimum, as an ideal $S$ using updated probabilities, perform diagnostic searches yoked with each $S$.

Number of Tests and D

The number of tests performed by Ss (NTS) totaled 67,327 . The number performed by the yoked optimum (NTO) totaled 59,103. Mean NTS per $\mathrm{D}=1,923.63$ and mean NTO per $\mathrm{D}=$ $1,688.66$. The ratio of total NTS to total NTO $=1.14$, indicating that, statistically, the optimum performed $14 \%$ better than Ss. Individual S-D ratios ranged from .95 (one $S$ performed better than the optimum on $\mathrm{D} 10$ ) to 1.32 , but the mean ratio per D varied from 1.02 to 1.10 , implying only a slight influence of D. No clear relationship between NTS and D moments, across complete Ds of 500 trials, was discernible.

However, regression analyses using NTS and NTO as criterion variables and Ex moments as predictors reveal substantial relationships that are primarily limited to MEx and VEx.

$$
\begin{aligned}
\mathrm{NTS}= & 1.53 \mathrm{MEx}+6.32 \mathrm{VEx}-.48 \mathrm{SEx} \\
& +.01 \mathrm{KEx}+124.86 \\
\mathrm{R}= & .78 \\
\mathrm{SE}= & 18.98 \\
\mathrm{NTO}= & .62 \mathrm{MEx}+5.81 \mathrm{VEx}-.18 \mathrm{SEx} \\
& -.01 \mathrm{KEx}+117.60 \\
\mathrm{R}= & .88 \\
\mathrm{SE}= & 10.33
\end{aligned}
$$

Not unexpectedly, because VEx determines the "spread" of component failures for a given $\mathrm{D}, \mathrm{VEx}$ is the largest contributor to NTS and NTO. The analysis also suggests that the optimum itself might be a predictor of Ss' performance. Bivariate regression analysis yielded the following result.

$$
\begin{aligned}
\mathrm{NTS} & =1.13 \mathrm{NTO}-2.22 ; \\
\mathrm{r} & =.82 ; \\
\mathrm{SE} & =17.37
\end{aligned}
$$

Although NTS is suboptimum, NTO and NTS are related by intervening factors. The same factors which influence NTO influence NTS, and nearly to the same degree.

\section{Search Structure}

Analysis of search structure was accomplished using "test trees" based on test sequences. For each $D$ and order of failures within $\mathrm{D}$, an optimal distribution of first tests (T1) develops over trials. Two optimal distributions of second tests, depending on whether T1 passes (P1) or fails (F1), will develop. Thus, test trees expand adding vertices (distributions of tests) that are each dependent upon outcomes of previous tests. Correspondingly, Ss' test trees may be compared to optimal test trees.

Optimal and $S$ test trees were obtained for each S-D combination averaged for Ss and optimum. These indicated that Ss' test trees resemble optimal solutions; however, as with Pc, Ss' search structure exhibits certain characteristics. For example, Ss' MT1 is displaced, relative to the optimum, away from component scale extremes, as Pc tends to be. Exceptions were D1, with Ss' MT1 equal to the optimum's, and D6. As noted earlier, the influence of D6's central placement is difficult to interpret.

Ss' range of tests at the third test level $($ range $=$ MP1P2-MF1F2) is also, on the average, $8 \%$ less than the optimal range in most cases. (This excludes $\mathrm{D} 1$ and $\mathrm{D} 10$. The optimum completed branches within two tests for these Ds.) D6 is again the exception, with Ss' test range exceeding the optimum by $21 \%$. This result is comparable to the one obtained above, showing VPc to be less than VEx except for D6.

A depressed P1 vertex or regression of MP1 away from sets of unresolved components is also a characteristic of the trees. Exceptions were D6 without a depression and D10, which has a depression in the opposite direction at F1 instead of $P 1$. This result may indicate that $\mathrm{Ss}$ are conservative when required to perform a second-level test in an unresolved set of components.
Of $245 \mathrm{~S}$ test distributions (to the third test level), the variance of 227 (93\%) exceeded optimal variance. Variance ratios (F) indicated that the combined variance of Ss' tests exceeded that of the optimum by a factor greater than 6 to $1(F=6.18)$.

At each test vertex, Ss' P1 $(F=11.62)$ and P1P2 $(F=11.00)$ distributions were more variable relative to the optimum than corresponding $\mathrm{F} 1(\mathrm{~F}=1.75)$ and $\mathrm{F} 1 \mathrm{~F} 2$ $(F=6.48)$ distributions. Comparing only $S$ test distributions at each test level, variances at pass vertices ( $P 1$, P1P2, F1P2) were larger than variances at corresponding fail vertices (F1, F1F2, P1F2).

These differences in test variability depending on test outcomes may result from a combination of the decreased range and depressed $\mathrm{P} 1$ vertex in Ss' search structure noted above. In other words, Ss generally tested conservatively into each unresolved set of components by small test increments. This produced large test variability after passes. Once the fail bracket (i.e., a test 'failed) was established, however, only a few possible tests remained in the resulting unresolved set, thereby restricting the size of the test variance after a test failed.

Although Ss' search structure is suboptimum, it does appear to be statistically logical. This statement is based principally on analyses indicating that NTS is predictable and that Ss' search structure generally matches optimal search structure. Suboptimal performance may be due to any or all of several characteristics of search structure, such as displaced MT1, inappropriate estimations of VEx magnitude, excessive test variabilities, and test variabilities that are differentially affected by pass or fail outcomes.

Comparison of NTS and Pc

A multiple regression analysis performed between NTS and Pc moments yielded the following equation, $R$, and $S E$ values.

$$
\begin{aligned}
\mathrm{NTS}= & .91 \mathrm{MPc}+5.01 \mathrm{VPc}+.28 \mathrm{SPc} \\
& -.05 \mathrm{KPc}+148.43 \\
\mathrm{R}= & .60 \\
\mathrm{SE}= & 24.23
\end{aligned}
$$

When compared with the NTS-Ex equation presented earlier, NTS, although related to $\mathrm{Pc}$, relies on Ex to a greater extent than Pc. Stated differently, D provides better prediction of NTS than subjective $D$ as reflected by $\mathrm{Pc}$.

It may be concluded that performance on the multiple response 
prediction and search tasks of this study is substantially influenced by varying probability distributions. There is, furthermore, some evidence that both prediction and diagnostic search exhibit certain characteristics, some of which may be common to both tasks. However, the exact nature of these characteristics and how they relate to such variables as the number of response alternatives and D positioning within these alternatives require more specific examination in future investigations.

\section{REFERENCES}

MILLS, R. G.: \& BAUER, $M_{0}$ A. WEIBD-WEIBG: Two programs for generating distributed stimulus events with specified moments. Behavioral Science, $1971,16,183$.

PETERSON, C. R., \& BEACH, L. R. Man as an intuitive statistician. Psychological Bulletin, $1967,68,29-46$. NOTE

1. This study has yielded extensive results which, because of limited space, cannot be presented here. A more detailed report will be available at a later date in the form of an AMRL technical report.

\section{CURRENT LITERATURE ON ATTENTION}

BAUMSTIMLER, Y., \& PARROT, J. (Université Laval, Cité Universitaire, Québec $10 \dot{e}$, P.Q., Canada). Stimulus generalization and spontaneous blinking in man involved in a voluntary activity. Journal of Experimental Psychology, 1971, 88, 95-102.

BERNSTEIN, A. S. (Department of Psychiatry, State University of New York, Downstate Medical Center, Brooklyn, N.Y. 11203), TAYLOR, K., AUSTEN, B. G., NATHANSON, M. \& 'SCARPELLI, A. Orienting response and apparent movement toward or away from the observer. Journal of Experimental Psychology, 1971, 87, 37-45.

DAS, J. P. (Centre for the Study of Mental Retardation, University of Alberta, Edmonton, Canada), \& BOWER, A. C. Orientating responses of mentally retarded and normal subjects to . word-signals. British Journal of Psychology, 1971, 62, 89-96.

GIAMBRA, L. M. (Miami University, Oxford, Ohio 45056). Selection strategies for eight concept rules with nonexemplar start cards. Journal of Experimental Psychology, 1971, 87, 78-92.

HIGGINS, J. D. (Department of
Psychiatry, Harvard University Medical School, Boston, Mass. 02115). Set and uncertainty as factors influencing anticipatory cardiovascular responding in humans. Journal of Comparative and Physiological Psychology, 1971, $74,272-283$.

KLATZKY, R. L., JUOLA, J. F., \& ATKINSON, R. C. (Stanford University, Stanford, Calif. 94305). Journal of Experimental Psychology, 1971, 87, 281-288.

MALTZMAN, I. (University of California, Los Angeles, Calif. 90024), HARRIS, L., INGRAM, E., \& WOLFF, C. A primacy effect in the orienting reflex to stimulus change. Journal of Experimental Psychology, 1971, 87, 202-206.

SMITH, M. C. (University of Toronto, Toronto 181, Ontario, Canada), \& RAMUNAS, S. Elimination of visual field effects by use of a single report technique: Evidence for order-of-report artifact. Journal of Experimental Psychology, 1971, 87, 23-28.

SMITH, R. E. (University of Washington, Seattle, Wash. 98105). Observational learning of modeled responses under shock-avoidance conditions as a function of attitude similarity and attraction toward the model. 1971, 22, 123-124.

SNAPPER, K. J. (Engineering Psychology Laboratory, Institute of Science and Technology, University of Michigan, Ann Arbor, Mich. 48105), \& FRYBACK, D. G. Inferences based on unreliable reports. Journal of Experimental Psychology, 1971, 87, 401-404.

WARM, J. S. (University of Cincinnati, Cincinnati, Ohio 45221), \& ALLUISI, E. A. Influence of temporal uncertainty and sensory modality of signals on watchkeeping performance. Journal of Experimental Psychology, 1971, 87, 303-308.

WITTER, D. W., MUELLER, J. H., \& MARX, M. H. (University of Missouri, Columbia, Mo. 65201). Correction procedures in observational learning. Psychonomic Science, 1971, 22, 94-95.

ZELNIKER, T. (Tel-Aviv University, Ramat-Aviv, Tel-Aviv, Israel). Perceptual attenuation of an irrelevant auditory verbal input as measured by an involuntary verbal response in a selective-attention task. Journal of Experimental Psychology, 1971, 87, 52-56. 